\title{
Importância do cirurgião-dentista no diagnóstico da bulimia: Revisão de literatura
}

\author{
Importance of dentist surgery in bulimia diagnosis: Literature review
}

Importancia del cirugía-dentista en el diagnóstico de bulimia:Revisión de literatura

Andressa Thaynara Andrade Gomes ${ }^{1}$, Kaique de Freitas Matos ${ }^{1}$, Luciana Quesado de Lavor ${ }^{1}$, Luiz Felipe Sampaio Pereira ${ }^{1}$, Yngrid Viviane Gomes de Macedo ${ }^{1}$, João Lucas de Sena Cavalcante ${ }^{1}$, Fátima Kateline Sampaio Pereira ${ }^{1}$, Letícia Tayná Bezerra Freire ${ }^{1}$, Williany Soares Damaceno ${ }^{1}$, Laiza Miranda Vasconcelos ${ }^{1}$, Victor Hudson Pereira Quirino ${ }^{1}$, Bruna Raissa Goncalves Moreira ${ }^{1}$, Alessandra Albuquerque Tavares Carvalho ${ }^{2}$, Marcília Ribeiro Paulino ${ }^{1}$, Natasha Muniz Fontes ${ }^{1 *}$.

\section{RESUMO}

Objetivo: Analisar através de uma revisão de literatura as manifestações orais mais comuns da bulimia. Métodos: Foi realizada a busca eletrônica de publicações nas bases de dados Scielo, PubMed e Google Acadêmico utilizando-se as seguintes palavras-chave: bulimia, bulimia nervosa dental erosion e oral manifestations. Foram adotados como critérios de inclusão dos estudos: a) artigos sobre a bulimia nervosa e o diagnóstico do cirurgião-dentista, b) artigos divulgados no período de janeiro de 2006 a fevereiro de 2018 e c) artigos publicados na língua inglesa. Segundo os critérios de inclusão e exclusão, realizou-se então a seleção de 30 trabalhos. Após sua análise, 10 artigos foram selecionados para o presente estudo. Resultados: Mesmo com o fácil acesso a veículos informativos atualmente, ainda há um baixo percentual de dentistas que fazem uma avaliação e tratamento voltados para transtornos alimentares como a bulimia, com alterações bucais presentes como erosão, carie dentária e xerostomia. Considerações finais: $O$ cirurgiãodentista deve detectar e tratar de forma específica as alterações bucais nos pacientes com bulimia, além de compreender as alterações orais de pacientes com transtornos alimentares e saber instruir esses pacientes após a vomito para reduzir os efeitos prejudiciais na cavidade oral.

Palavras-chave: Bulimia, Bulimia Nervosa, Erosão Dentária, Manifestações Bucais.

\section{ABSTRACT}

Objective: To analyze the most common oral manifestations of bulimia through a literature review. Methods: The electronic search of publications in the databases Scielo, PubMed and Google Scholar was performed using the following keywords: bulimia, bulimia nervosa dental erosion and oral manifestations. The following were adopted as inclusion criteria: a) articles on bulimia nervosa and the diagnosis of the dentist, b) articles published from January 2006 to February 2018 and c) articles published in English. According to the inclusion and exclusion criteria, 30 papers were selected. After its analysis, 10 articles were selected for the present study. Results: Even with the easy access to informational vehicles currently, there is still a low percentage of dentists who make an evaluation and treatment for eating disorders such as bulimia, with oral disorders present as erosion, dental caries and xerostomia. Final considerations: The surgeon-dentist should detect and treat specific oral alterations in patients with bulimia, in addition to understanding the oral alterations of patients with eating disorders and to instruct these patients after vomiting to reduce the harmful effects in the oral cavity.

Key words: Bulimia, Bulimia Nervosa, Tooth Erosion, Oral Manifestations.

${ }^{1}$ Centro Universitário Dr. Leão Sampaio - Juazeiro do Norte - Ceará. *E-mail: natashafontes@hotmail.com 2Universidade Federal de Pernambuco, Recife, PE, Brasil. 


\section{RESUMEN}

Objetivo: Analizar través de una revisión las manifestaciones orales más comunes de la bulimia. Métodos: Se realizó la búsqueda electrónica de publicaciones en las bases de datos Scielo, PubMed y Google Académico utilizando las siguientes palabras clave: bulimia, bulimia nerviosa dental erosión y oral manifestaciones. Se han adoptado como criterios de inclusión de los estudios: a) artículos sobre la bulimia nerviosa y el diagnóstico del cirujano-dentista, b) artículos divulgados en el período de enero de 2006 a febrero de 2018 y c) artículos publicados en la lengua inglesa. Según los criterios de inclusión y exclusión, se realizó la selección de 30 trabajos. Después de su análisis, 10 artículos fueron seleccionados para el estudio. Resultados: Incluso con el fácil acceso a vehículos informativos actualmente, todavía hay un bajo porcentual de dentistas que hacen una evaluación y tratamiento dirigidos a trastornos alimentarios como la bulimia, con alteraciones bucales presentes como erosión, carie dental y xerostomía. Consideraciones finales: El cirujano-dentista debe detectar y tratar de forma específica las alteraciones bucales en los pacientes con bulimia, además de comprender las alteraciones orales de pacientes con trastornos alimentarios y saber instruir a esos pacientes después del vómito para reducir los efectos perjudiciales en la cavidad oral.

Palabras clave: Bulimia, Bulimia Nerviosa, Erosión Dentaria, Manifestaciones Bucales.

\section{INTRODUÇÃO}

A bulimia nervosa é um transtorno alimentar caracterizado pela ingestão de uma grande quantidade de alimentos hipercalóricos em um curto período de tempo, e logo após o paciente usa de métodos compensatórios inadequados para evitar o ganho de peso (ARANHA ACC, et al., 2008). Dentre os métodos compensatórios utilizados estão o uso de laxantes, a prática excessiva de exercícios físicos e o vômito auto induzido, além de cerca de $5 \%$ da população total de brasileiros apresentar esse transtorno algum momento da vida (ROMANOS GE, et al., 2012). As manifestações orais mais comuns causadas pelos vômitos frequentemente induzidos, são as erosões dentárias (ARANHA ACC, et al., 2008).

A erosão dentária é uma patologia que remove o esmalte dental através de um processo químico. Tal manifestação pode se agravar em decorrência da presença de alguns fatores como a quantidade de purgas por dia, duração, os hábitos de higiene bucal do paciente, se há enxague após o vômito e se o mesmo usa de substâncias neutralizantes como o leite (ROMANOS GE, et al., 2012).

A perimilólise é um tipo específico de erosão dental que é causada por fatores químicos como a regurgitação do conteúdo gástrico, e mecânicos, como a ativação dessas lesões pelos movimentos linguais logo em seguida. As lesões geralmente se localizam na região palatina de dentes anteriores e tem um aspecto brilhante e liso (ARANHA ACC, et al., 2008). Após dois anos de hábitos purgativos regulares, é que as lesões estão evidentes para serem inspecionadas (BERN EM et al., 2016).

Alguns estudos enfatizaram a importância desses aspectos que se manifestam clinicamente na cavidade oral, na realização de um diagnóstico diferencial pelo cirurgião dentista em pacientes com distúrbios alimentares (ROMANOS GE, et al., 2012). Segundo Bern EM et al. (2016), é possível distinguir as lesões causadas pelo refluxo gastroesofágico crônico das causadas pela bulimia, já que no primeiro, a erosão está presente nas superfícies linguais e oclusais dos dentes posteriores. Além disso, há o aumento não inflamatório das glândulas salivares, também conhecido como sialadenite, esse aumento acomete principalmente a parótida, em decorrência do comportamento repetitivo de purga. Esse aumento pode ser simétrico ou unilateral e é muitas vezes um dos responsáveis por fazer com que o paciente busque cuidados médicos devido a sua preocupação com a estética facial (BERN EM et al., 2016).

Existem diversas maneiras de prevenir os transtornos alimentares, essas podem ser primárias, que consistem em impedir que o problema de saúde ocorra, e, a prevenção secundária que é composta de atividades que permitam a detecção precoce das manifestações da doença, mudando a gravidade e extensão da mesma (DEBATE RD e TEDESCO LA, 2006). 
Diante disso, o objetivo do presente estudo é familiarizar o cirurgião dentista com as manifestações orais da bulimia para que esse consiga fazer um diagnóstico precoce da mesma, e saiba orientar o paciente em relação aos cuidados com sua higiene oral evitando um agravo das lesões dentárias.

\section{MÉTODOS}

Foi realizada a busca eletrônica de publicações nas bases de dados Scielo, PubMed e Google Acadêmico utilizando-se as seguintes palavras-chave, obtidas de acordo com o Medical Subject Headings (MeSH): bulimia, bulimia nervosa, erosão dentária e manifestações bucais, usando o operador booleano "AND". Foram adotados como critérios de inclusão dos estudos: a) artigos sobre a bulimia nervosa e o diagnóstico do cirurgião dentista, b) artigos divulgados no período de janeiro de 2006 a fevereiro de 2018 e c) artigos publicados na língua inglesa. Segundo os critérios de inclusão e exclusão, realizou-se então a seleção de 30 trabalhos. Após sua análise, 20 foram excluídos e, ao final, 10 artigos foram selecionados para o presente estudo (Figura 1).

Figura 1 - Fluxograma de pesquisa bibliográfica.
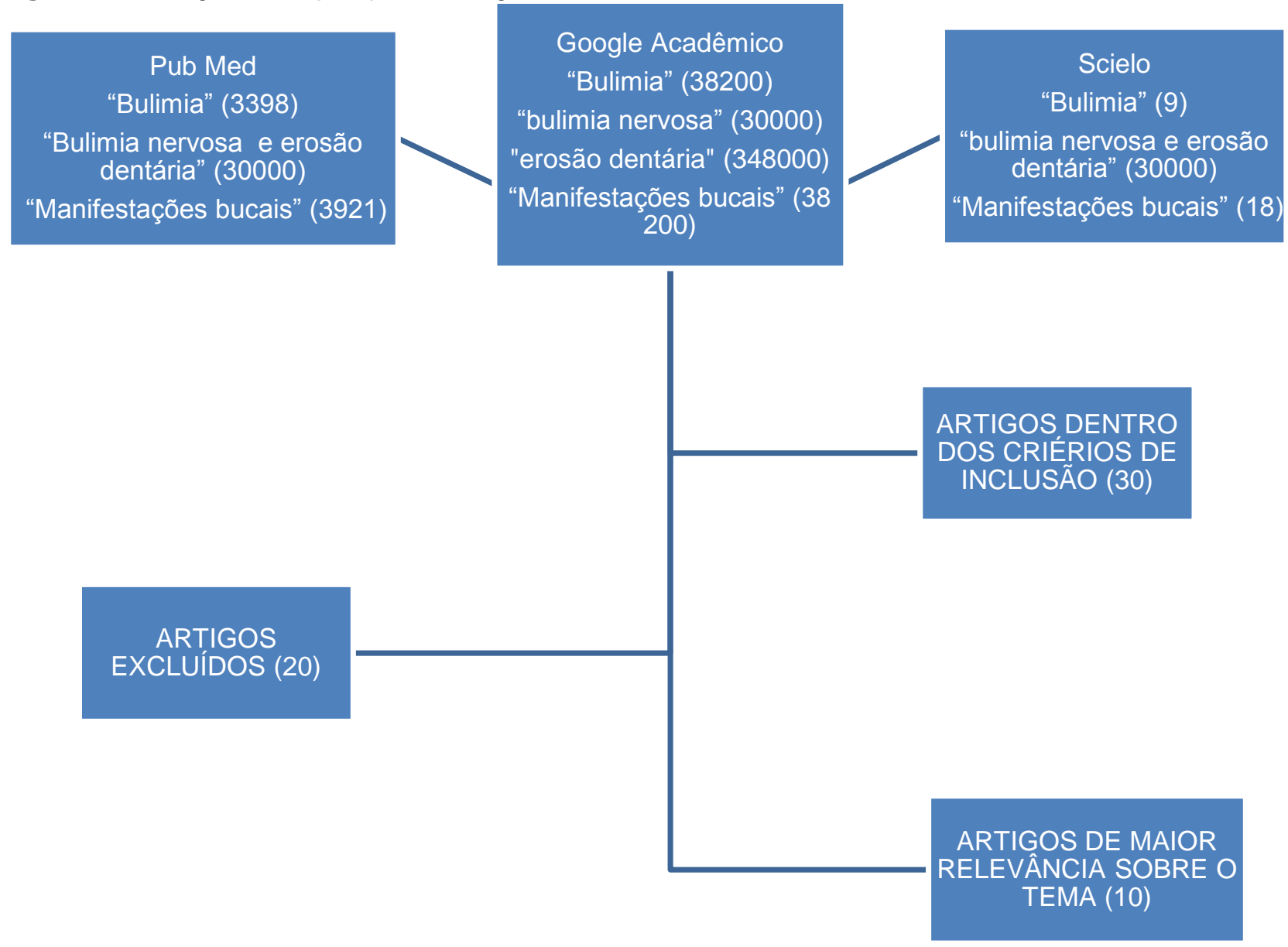

Fonte: Dados de pesquisa, 2019.

\section{RESULTADOS}

De acordo com os dados analisados na literatura iremos apresentar em forma de tabela os achados acerca da associação da bulimia e as condições bucais para analisar a importância do cirurgião-dentista sobre seu diagnostico (Tabela 1). 
Tabela 1 - Resultados bibliográficos da pesquisa.

\begin{tabular}{|c|c|c|c|}
\hline AUTOR-ANO & TIPO DE ESTUDO & METODOLOGIA & RESULTADOS \\
\hline $\begin{array}{l}\text { ARANHA ACC, } \\
\text { et al., } 2008\end{array}$ & Revisão sistemática & $\begin{array}{l}\text { Foi realizada um a revisão de literatura destacando as } \\
\text { consequências orais dos transtornos alimentares } \\
\text { (anorexia e bulimia) }\end{array}$ & $\begin{array}{l}\text { O estudo revela que algumas manifestações } \\
\text { estão presentes em pessoas com distúrbios } \\
\text { alimentares, são elas, erosão dentária, } \\
\text { xerostomia, aumento da glândula parótida } \\
\text { entre outras. }\end{array}$ \\
\hline $\begin{array}{l}\text { BERN EM, et } \\
\text { al.,2016 }\end{array}$ & $\begin{array}{l}\text { Revisão de literatura } \\
\text { (Manual de } \\
\text { Alterações) }\end{array}$ & $\begin{array}{l}\text { Revisão dos critérios diagnósticos para transtornos } \\
\text { alimentares, na tentativa de melhor caracterizar os } \\
\text { indivíduos com relação patológica com alimentos. Os } \\
\text { transtornos alimentares agora incluem não apenas } \\
\text { anorexia nervosa, bulimia nervosa e transtorno da } \\
\text { compulsão alimentar periódica, mas também, ruminação } \\
\text { e transtorno alimentar evitante / restritivo. }\end{array}$ & $\begin{array}{l}\text { Indivíduos com anorexia e bulimia, podem } \\
\text { apresentar uma gama de manifestações. A } \\
\text { cavidade oral, as glândulas salivares, o trato } \\
\text { gastrointestinal, o pâncreas e o fígado } \\
\text { podem ser afetados por comportamentos } \\
\text { restritivos nutricionais e compulsivos / } \\
\text { purgativos. As complicações são } \\
\text { frequentemente reversíveis com terapia } \\
\text { nutricional apropriada. }\end{array}$ \\
\hline $\begin{array}{l}\text { CONVISER JH, } \\
\text { et al., } 2014\end{array}$ & Estudo transversal & $\begin{array}{l}\text { Foram selecionadas } 292 \text { mulheres maiores de } 18 \text { anos } \\
\text { que estavam em centros de tratamento e com } \\
\text { diagnóstico de bulimia para avaliar os cuidados } \\
\text { realizados pelas mesmas após comportamentos } \\
\text { purgativos, as complicações dentárias presentes e } \\
\text { relação comunicativa com seus dentistas. }\end{array}$ & $\begin{array}{l}\text { Aproximadamente } 92 \text { por cento das } \\
\text { mulheres participantes admitiram possuir } \\
\text { complicações dentárias, 32,5 por cento } \\
\text { mencionaram que fazem escovação } \\
\text { imediatamente após vomitar e somente } 29,2 \\
\text { por cento dessas mulheres falaram sobre } \\
\text { bulimia com o cirurgião dentista. }\end{array}$ \\
\hline $\begin{array}{l}\text { DEBATE RD, } \\
\text { et al., } 2007\end{array}$ & Estudo transversal & $\begin{array}{l}\text { Foi aplicado um questionário para } 316 \text { participantes com } \\
\text { perguntas relacionadas a cuidados específicos para } \\
\text { transtornos alimentares. }\end{array}$ & $\begin{array}{l}\text { Os resultados mostraram ausência de } \\
\text { cooperação entre o cirurgião dentista e } \\
\text { outros profissionais da área da saúde que } \\
\text { propiciam tratamento para pacientes } \\
\text { diagnosticados com algum transtorno } \\
\text { alimentar, e pouca capacitação dos dentistas } \\
\text { pelos programas de saúde bucal. }\end{array}$ \\
\hline $\begin{array}{l}\text { DEBATE RD e } \\
\text { TEDESCO LA, } \\
\quad 2006\end{array}$ & Pesquisa qualitative & $\begin{array}{l}\text { Foi realizada uma avaliação exploratória entre cirurgiões } \\
\text { dentistas sobre prevenção secundária nos distúrbios } \\
\text { alimentares. }\end{array}$ & $\begin{array}{l}\text { Os resultados deste estudo indicam que há } \\
\text { uma necessidade de treinamento adequado } \\
\text { para preparar melhor os profissionais de } \\
\text { saúde bucal para o atendimento integral ao } \\
\text { paciente. }\end{array}$ \\
\hline
\end{tabular}

$\overline{\text { REAS/EJCH | Vol. Sup. } 26 \text { | e730 | DOI: https://doi.org/10.25248/reas.e730.2019 Página } 4 \text { de } 8}$ 


\begin{tabular}{|c|c|c|c|}
\hline $\begin{array}{l}\text { DYNESEN AW, } \\
\text { et al., } 2008\end{array}$ & $\begin{array}{l}\text { Estudo de caso } \\
\text { controle }\end{array}$ & $\begin{array}{l}\text { Foram selecionadas vinte mulheres com bulimia e vinte } \\
\text { para grupo controle para análise salivar em relação a } \\
\text { quantidade, composição e sensação de boca seca } \\
\text { relatada por elas. }\end{array}$ & $\begin{array}{l}\text { Houve redução da quantidade de saliva não } \\
\text { estimulada nas pacientes com bulimia } \\
\text { quando comparadas ao grupo controle, já no } \\
\text { fluxo salivar estimulado não houve } \\
\text { diferenças significativas. }\end{array}$ \\
\hline $\begin{array}{c}\text { ROMANOS } \\
\text { GE, et al., } 2012\end{array}$ & Revisão de literatura & $\begin{array}{l}\text { Foi feito uma revisão de literatura para analisar as } \\
\text { manifestações orais mais comuns em paciente com } \\
\text { transtornos alimentares. }\end{array}$ & $\begin{array}{l}\text { Em geral, manifestações como aumento da } \\
\text { glândula parótida, lábios ressecados entre } \\
\text { outros, são comuns em pacientes com } \\
\text { distúrbio alimentar. }\end{array}$ \\
\hline $\begin{array}{l}\text { RUSSO L, et } \\
\text { al., } 2007\end{array}$ & Revisão crítica & $\begin{array}{l}\text { Analisar criticamente as manifestações orais, } \\
\text { prognóstico e conduta das pessoas com transtornos } \\
\text { alimentares. }\end{array}$ & $\begin{array}{l}\text { As manifestações dos distúrbios envolvem } \\
\text { uma série de estruturas bucais e também } \\
\text { faciais como periodonto, glândulas e tecidos } \\
\text { periorais. Essas manifestações são } \\
\text { causadas por uma série de fatores } \\
\text { relacionados ao distúrbio como drogas, } \\
\text { deficiência nutricional má higiene pessoal, } \\
\text { entre outros. }\end{array}$ \\
\hline $\begin{array}{l}\text { VIVEK HP, et } \\
\text { al., } 2018\end{array}$ & Estudo In Vitro & $\begin{array}{l}\text { Foi realizado um estudo com espécimes de esmalte que } \\
\text { foram divididos em três grupos, para logo após serem } \\
\text { expostos a três tipos de substâncias durante um } \\
\text { determinado espaço de tempo. Essas substâncias era o } \\
\text { azeite de oliva a } 2 \text { por cento, enxaguatório bucal com } \\
\text { azeite de oliva a } 2 \text { por cento (Xerostom), e ao } \\
\text { enxaguatório Listerine. A remineralização resulta na } \\
\text { exposição durante } 5 \text { minutos as três substâncias, e } \\
\text { posteriormente ao ácido cítrico durante } 3 \text { minutos. }\end{array}$ & $\begin{array}{l}\text { Listerine e Xerostom foram as duas } \\
\text { substâncias que mostraram resultados } \\
\text { relevantes na capacidade de proteção } \\
\text { antierosiva e diminuição do efeito erosivo. O } \\
\text { azeite de oliva a } 2 \text { por cento apresentou } \\
\text { menor proteção. }\end{array}$ \\
\hline $\begin{array}{c}\text { LOURENÇO } \\
\text { M, et al., } 2018\end{array}$ & $\begin{array}{l}\text { Estudo de caso } \\
\text { controle }\end{array}$ & $\begin{array}{l}\text { Foram selecionadas } 55 \text { mulheres com diagnóstico de } \\
\text { anorexia ou bulimia que estavam em tratamento do } \\
\text { ambulatório do Centro Hospitalar São João, onde } 33 \\
\text { participaram da pesquisa. Foi executada a aplicação de } \\
\text { questionários e realização de exames clínicos orais. }\end{array}$ & $\begin{array}{l}\text { Os resultados mostraram uma maior } \\
\text { possibilidade de desenvolvimento de cárie, } \\
\text { erosão dentária, alterações salivares e } \\
\text { periodontais nos pacientes com distúrbios } \\
\text { alimentares do que em pacientes que não } \\
\text { tem o transtorno. }\end{array}$ \\
\hline
\end{tabular}

Fonte: Dados da pesquisa, 2019.

REAS/EJCH | Vol. Sup. 26 | e730 | DOI: https://doi.org/10.25248/reas.e730.2019 Página 5 de 8 


\section{DISCUSSÃO}

Os transtornos alimentares apresentam manifestações comuns na cavidade oral, tais manifestações são decorrentes de vômitos auto induzidos e deficiências nutricionais, podendo afetar glândulas salivares, cavidade oral, tecido perioral e dentes. O uso de medicamentos, a falta de higiene pessoal e hábitos alimentares anormais podem aumentar os sintomas de tais (BERN EM et al., 2016). Adolescentes do sexo feminino e atletas e/ou bailarinos são destacados nos países ocidentalizados como sendo mais susceptíveis a desenvolverem o transtorno bulímico, já que tais grupos destacam a magreza como necessária para uma boa imagem ou boa execução das atividades (DYNESEN AW, et al., 2008).

Dentre as diversas manifestações orais causadas pelas exposições ácidas, destaca-se a erosão dental, sensibilidade dental, xerostomia e aumento nas taxas de cárie e doença periodontal. Percebe-se também, manifestações extra orais, como o aumento da glândula parótida (CONVISER JH, et al., 2014). Os pacientes com transtornos alimentares apresentam alterações mais comuns quando comparados indivíduos saudáveis, essas podem ser lábios secos ou rachados, sensação de queimação na boca ou principalmente na língua, e efeitos sobre glândulas salivares e secreção salivar (ROMANOS GE, et al., 2012).

Apesar dos pacientes com distúrbios alimentares apresentarem inferioridade em relação a saúde bucal quando comparados a indivíduos saudáveis organizados por idade e sexo, os parâmetros de identificação de tal transtorno como cárie dentária, aumento da glândula parótida, hipossalivação, abrasão dentária, hipersensibilidade, dentre outros, mostram com muita frequência nestes pacientes com transtornos alimentares (LOURENÇO M, et al., 2018).

Pacientes bulímicos que tem uma dieta com baixo teor de ácido, exibem inicialmente erosões nas superfícies oclusais e palatinas dos dentes superiores. Alguns estudos dão ênfase na localização da desmineralização do esmalte para auxiliarem na realização de um diagnóstico diferencial em pacientes com distúrbios alimentares (ROMANOS GE, et al., 2012). Perimilólise é um tipo específico de erosão do esmalte, que tem fatores químicos e mecânicos, causados pela regurgitação do conteúdo gástrico e ativados pelos movimentos linguais logo após. Essas lesões tem um aspecto liso e brilhante e geralmente se manifestam nas superfícies palatinas dos dentes anteriores superiores (ARANHA ACC, et al., 2008).

Estudos mostraram que pacientes que tem transtorno alimentar com vômitos auto induzidos apresentaram mais erosão dentária, do que os pacientes com transtornos alimentares sem o comportamento de purga (CONVISER JH, et al., 2014). Erosões dentárias podem ser percebidas após aproximadamente dois anos ao início das práticas de vômitos frequentes (BERN EM et al., 2016). Substâncias que ajudem a prevenir a desmineralização e que auxiliem na remineralização é um fator relevante quando se trata de erosão dentária. Em um estudo in vitro realizado por Vivek HP, et al. (2018), com 45 espécimes que foram divididos em três grupos e cada um submetido a exposição com um tipo de substância.

O primeiro foi exposto ao Xerostom, enxaguatório bucal contendo $2 \%$ de azeite de oliva, o segundo grupo ao azeite de oliva a $2 \%$ e o terceiro a um enxaguatório bucal fluoretado (listerine). Os resultados desse estudo mostraram que o Xerostom e o Listerine além de serem antierosivos, diminuem ainda o efeito erosivo no esmalte, sendo os dois equivalentes nos resultados. O azeite a $2 \%$ mostrou menor potencial de ação nos dois casos citados (LOURENÇO M, et al., 2018).

Em relação a maior susceptibilidade a cárie em pacientes com distúrbios alimentares, ainda há conflito quando se diz respeito aos resultados. Sabe-se que pessoas com distúrbios alimentares consomem uma dieta rica em açúcares várias vezes por dia, podendo ser uma fonte de nutrição para as bactérias cariogênicas residentes na cavidade oral. $\mathrm{O}$ uso de medicamentos antidepressivos por alguns pacientes podem causar xerostomia, que por sua vez associada a dieta cariogênica e a deficiente higienização cria um ambiente propício para o acúmulo de placa nos dentes e multiplicação dos microrganismos (ROMANOS GE, et al., 2012). A doença periodontal é uma doença inflamatória que afeta os tecidos de suporte do dente como gengiva, ligamento periodontal, cimento e osso alveolar. O fator de risco mais comum em tal, é a fraca manutenção da higiene oral. É uma manifestação pouco comum em indivíduos com distúrbios alimentares já que a maioria são jovens e a doença está relacionada ao avanço da idade (ROMANOS GE, et al., 2012). Em 
crianças, inflamações gengivais e periodontites que apresentem dor e vermelhidão, devem ser incluídas no diagnóstico diferencial, se acompanhadas de algum transtorno alimentar, uma vez que, tais aparições são consideradas incomuns em crianças. Comportamentos de purga podem causar eritema palatino e úlceras, principalmente no palato mole, devido a exposição a ácido crônico e ao trauma repetitivo por indução digital (BERN EM et al., 2016).

Os pacientes que sofrem com esse distúrbio alimentar, e provocam vômito, geralmente apresentam aumento nas glândulas parótidas. Quanto a sua ocorrência e extensão, são diretamente proporcionais à duração e severidade dos vômitos autoinduzidos. Estimou-se que o inchaço da glândula ocorre entre 10-50\% dos casos, podendo ser ainda, unilateral ou bilateral (ARANHA ACC, et al., 2008). Conviser JH, et al. (2014) enfatiza o quão é importante o diálogo entre o cirurgião-dentista e o paciente, pois essa comunicação tem influência clínica. Mostrar familiarização quando se diz respeito a transtornos alimentares por parte do profissional, disponibilizar pequenos questionários sobre este tópico na sala de espera do seu consultório por exemplo, ajudam no encorajamento dos pacientes para buscar ajuda.

Embora haja um aumento, na porcentagem de programas de higiene dental e a inclusão dos distúrbios alimentares dentro dos currículos em relação ao ano passado, a carga horária dedicada a familiarização dos profissionais aos sinais apresentados nos transtornos alimentares é mínima, dificultando o atendimento integral aos pacientes portadores dos transtornos (DEBATE RD, et al., 2007).

Os distúrbios alimentares exigem uma avaliação abrangente, pois possuem manifestações que envolvem uma série de estruturas bucais e também faciais como periodonto, glândulas e tecidos periorais, além de um vínculo oral/sistêmico necessitam de um tratamento multidisciplinar. Torna-se necessária a educação dos cirurgiões-dentistas para que esses profissionais possam ter um melhor conhecimento no que se diz respeito a prevenção, reconhecimento precoce de doenças orais e assim, colaborar para o bom desempenho dos outros profissionais de saúde diante do tratamento desses distúrbios (DEBATE RD e TEDESCO LA, 2006; RUSSO L, et al., 2007). A influência do vômito sobre a saúde bucal e problemas relacionados a cavidade oral é enfoque em muitos estudos, onde as manifestações mais presentes em pessoas com distúrbios alimentares, são elas erosão dentária, xerostomia, aumento da glândula parótida entre outras (ARANHA ACC, et al., 2008).

O vômito e o surgimento da erosão dentária têm uma relação forte com lesões de comprometimento em dentina e esmalte, onde a maioria acomete em ambos tecidos dentinários. Como proteção erosiva, após o vômito é indicado o uso de algumas substâncias para como o Listerine e Xerostom na capacidade de proteção antierosiva e diminuição do efeito erosivo sobre a estrutura dentaria (LOURENÇO M, et al., 2015; VIVEK HP, et al., 2018). Já em relação ao desenvolvimento da cárie estudos demonstram que esses pacientes apresentam uma maior predisposição pois apresentam um elevado índice de CPOD (índice de dentes cariados, perdidos ou obturados) (ROMANO GE, et al., 2012; LOURENÇO M, et al., 2018).

Romanos GE, et al. (2012), relata que, pacientes com bulimia apresentam além dos vômitos frequentes, fatores associados ao desenvolvimento e predisposição a doença cárie, como: má higienização bucal, dieta cariogênica e uso de antidepressivos. Porém Lourenço $M$, et al. (2018), discorda quando se trata da higienização oral dos pacientes bulímicos, já que no seu estudo os mesmos apresentaram boa frequência na escovação e adequadas práticas após o vômito, como neutralização ácida com soluções adequadas ou água.

As glândulas salivares são um dos aspectos mais estudados nesta enfermidade, onde apresentam uma menor secreção de saliva devido ao uso de medicamentos e não uma alteração secretora nas glândulas salivares, sendo assim ocorre apenas uma redução do fluxo salivar não estimulado. $O$ fluxo salivar em paciente com bulimia independente do consumo ou não de medicamentos antidepressivos, ou seja, não apresentam taxas alteradas. A única alteração é da quantidade de saliva não estimulada (ARANHA ACC, et al., 2008; DYNESEN AW, et al., 2008).

Estudos sobre o correto diagnóstico e uma boa prevenção em consultas iniciais entres os cirurgiõesdentistas para pacientes portadores da bulimia, mostram que poucos dentistas estão aptos a realizar a prevenção secundária de distúrbios alimentares. Além de não relataram instruir o paciente com transtorno alimentar ao atendimento odontológico, indicando a escovação após o vômito, para redução os efeitos 
maléficos sobre a estrutura dentária. A avaliação de rotina do cirurgião-dentista em seu consultório requer uma analise minuciosa para observar os aspectos não somente de alteração estrutural no esmalte dentário do paciente portador de bulimia mais também a analise psicológica através de uma adequada anamnese do paciente para que assim seja feita o encaminhamento desses pacientes para um tratamento multidisciplinar com envolvimento medico, psicólogo e cirurgião-dentista (DEBATE RD e TEDESCO LA, 2006; DEBATE RD, et al., 2007; CONVISER JH, et al., 2014).

\section{CONSIDERAÇÕES FINAIS}

A bulimia acarreta diversas alterações na cavidade oral do paciente, muitas dessas manifestações são irreversíveis e quando se encontram em grau avançado prejudicam a qualidade de vida de quem tem ou teve o transtorno. A importância do cirurgião-dentista se familiarizar com as alterações presentes na cavidade oral é fazer um diagnóstico precoce possibilitando o tratamento multidisplinar entre os profissionais de saúde, evitando que o transtorno bulímico se agrave trazendo consequências irreversíveis.

\section{REFERÊNCIAS}

1. ARANHA ACC et al. Eating Disorders Part I: Psychiatric Diagnosis and Dental Implications. The Journal of Comtemporary Dental Practice. 2008; v. 9, n. 6, p. 1-11.

2. ROMANOS GE et al. Oro-facial manifestations in patients with eating disorders. Appetite. 2012; v. 59, p. $499-504$.

3. BERN EM et al. Gastrointestinal Manifestations of Eating Disorders. Journal of Pediatric Gastroenterology and Nutrition. 2016; v. 63, n. 5, p. 77-85.

4. DEBATE RD, Tedesco LA. Increasing Dentists Capacity for Secondary Prevention of Eating Disorders: Identification of Training, Network, and Professional Contingenciesn. Journal of Dental Education. 2006; v. 70, n. 10, p. $1066-1075$.

5. DYNENSEN AW et al. Salivary changes and dental erosion in bulimia nervosa. Oral Surg Oral Med Oral Pathol Oral Radiol Endod. 2008; v. 106, n. 5, p. 696-707.

6. CONVISER JH et al. Oral care behavior after purging in a sample of women with bulimia nervosa. JADA. 2014; v. 145, n. 4, p. 352-354.

7. LOURENÇO $\mathrm{M}$ et al. Orofacial manifestations in outpatients with anorexia nervosa and bulimia nervosa focusing on the vomiting behavior. Clinical Oral Investigations. 2018; 22(5):1915-1922.

8. VIVEK HP et al. Effect of Mouthrinses containing Olive Oil, Fluoride, and Their Combination on Enamel Erosion: An in vitro Study. The Journal of Contemporary Dental Practice. 2018; v. 19, n. 2, p.130-136.

9. DEBATE RD et al. Eating Disorders in the Oral Health Curriculum. Journal of Dental Education. 2007; v. 71, n. 5, p. 655-663.

10. RUSSO L et al. Oral manifestations of eating disorders: a critical review. Oral Diseases. 2007; v. 14, p. $479-484$. 